\title{
Ideal Technique of Incisional Hernia Repair: The Quest Continues
}

\author{
Debajyoti Mohanty • Anjay Kumar • \\ Ashwani Kumar Dalal
}

Received: 19 October 2013 / Accepted: 21 October 2013 /Published online: 6 November 2013

(C) Association of Surgeons of India 2013

We have read with interest the article "A comparative analysis on various techniques of incisional hernia repair-experience from a tertiary care teaching hospital in South India" by Kumar et al. [1]. We would like to complement the authors for managing such a large number of patients within a time span of 2 years and following them over a period of 5 years. We believe that certain aspects of this article need clarification: preperitoneal mesh placement was coined "onlay meshplasty" in the introduction but "underlay repair" in the comments section. Traditionally, an onlay repair denotes the site of implantation of the mesh on the anterior rectus sheath, inlay repair implies fixation of the mesh to the aponeurotic edges of the rectus sheath, and underlay or sublay repair means the mesh is placed in the retrorectus and preperitoneal space [2].

The patients were subjected to different techniques of repair irrespective of the size of hernia and followed up for 5 years as per the prospective study protocol; however, the analysis section mentioned that anatomical repair was attempted in patients with size of defect less than $5 \mathrm{~cm}$, while patients were followed up for a variable period of 2 24 months.

The authors had observed statistically significant difference in the intensity of postoperative pain and the rate of recurrence following hernia repair, but they were silent about the type of statistical test used and the level of significance considered for comparing the parameters. Chi-square and Fisher exact tests are the commonly used tests for proportions involving qualitative variables. The study had recorded that postoperative pain was more (VAS score $>5$ ) with underlay repair than onlay repair $(p<0.005)$ and anatomical repair $(p<0.001)$. Applying

D. Mohanty $(\varangle) \cdot$ A. Kumar $\cdot$ A. K. Dalal

Department of General Surgery, All India Institute of Medical Sciences, Raipur, India

e-mail: debajyoti.mohanty@gmail.com
Fisher exact test with a $p$ value of $<0.05$ taken as significant difference to the same set of observations revealed significant increase in pain intensity in underlay repair when compared to anatomical repair $(p=0.002)$, but not in relation to onlay repair $(p=0.052)$. Similarly in respect to the rate of recurrence following anatomical (6/21) and mesh repair (5/48), the difference was not found to be statistically significant with a $p$ value of 0.077 on Fisher exact test. This casts a doubt about the authors' proposition for repair of all incisional hernias with mesh reinforcement on the basis of their study.

Follow-up loss of $\geq 20 \%$ greatly reduces the validity of the observed results in a prospective study [3]. Loss to follow-up in this study stood at 21/90 (23.3\%) patients, and the recurrence rate was calculated in the remaining 69 patients. Since a total of 11/69 patients experienced hernia recurrence, the overall recurrence rate was $15.94 \%$ and not $12 \%$ as the authors had claimed.

Lastly, the ongoing quest for the ideal technique of incisional hernia repair in Indian scenario is still continuing. The breakthrough can only be achieved by a high quality randomized controlled trial having a large sample size from this part of the world.

\section{References}

1. Kumar V, Rodrigues G, Ravi C, Kumar S (2013) A comparative analysis on various techniques of incisional hernia repair-experience from a tertiary care teaching hospital in south India. Indian J Surg 75(4):271-273

2. de Vries Reilingh TS, van Geldere D, Langenhorst B, de Jong D, van der Wilt GJ, van Goor H, Bleichrodt RP (2004) Repair of large midline incisional hernias with polypropylene mesh: comparison of three operative techniques. Hernia 8(1):56-59

3. Sackett DL, Strauss SE, Richardson WS, Rosenberg WMC, Haynes RB (2000) Evidence based medicine: how to practice and teach EBM, 2nd edn. Churchill Livingstone, Edinburgh 